\title{
Investigation of the Behaviour of a Tilted Up Type Control System of a Small Wind Turbine
}

\section{Mahinsasa Narayana and Kapila Peiris}

Abstract: In small-scale wind turbines various types of control mechanisms are used to protect them from high wind speeds, i.e. when the wind speed exceeds the rated value. In tilted up type small-scale wind turbines the rotor is free to move around horizontal as well as vertical axes. Therefore when the wind speed exceeds the rated value the rotor tilts upwards and sideways and comes to a stable position with an inclination to the direction of wind and hence controlling the component of the wind responsible for power generation (and for the rotational speed of the rotor). This paper describes, how a mathematical model is developed by using $\mathrm{D}^{\prime}$ Alambert's principle to investigate the complex behavior of the wind turbine during the transient period and the stable position.

Keywords : Wind turbine, Rotor, Tail vane, D'Alambert's Principle

\section{Introduction}

Main task of a wind turbine is to convert wind power to electricity. Wind turbine must face itself into the wind and it has to adapt to given circumstances. It also needs to protect itself from the violence of winds greater than the rated wind speed this must happen automatically.

Larger wind turbines have computer driven control systems, which operate servomotors, hydraulic motors etc. Small wind turbines need simple passive controls wherever possible.

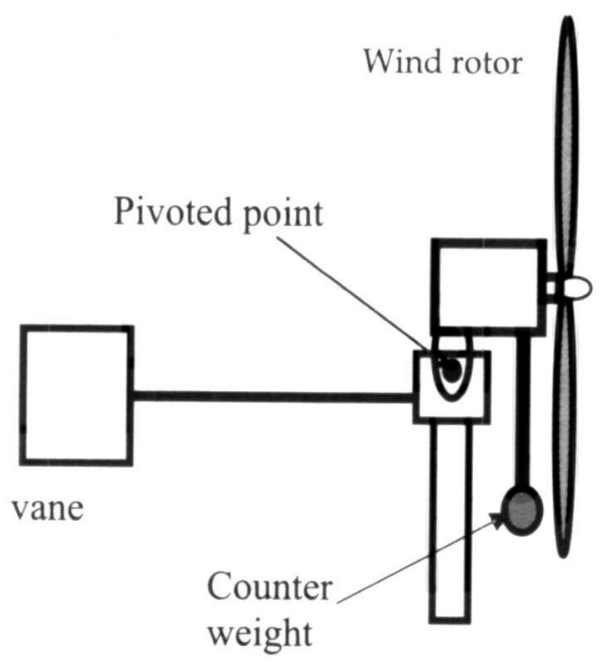

Figure 1 : Schematic diagram of tilt up type small wind turbine

Control systems of small-scale wind turbines are always simple. Tilt up method is a simple method to control the small-scale wind turbine. Figure 1 shows the kinematic details of the system. In this method, the rotor, generator and counter weights can be rotated freely around the pivot point as well as around the vertical axis (i.e. tower axis). Wind rotor is pivoted in vertical plane and it could tilt upward due to drag force of wind rotor. Drag force depends on the wind speed.

This paper describes a theoretical investigation of the control behaviour of a small wind turbine produced in Sri Lanka. A picture of this tilt up type small wind turbine is shown in figure 2 .

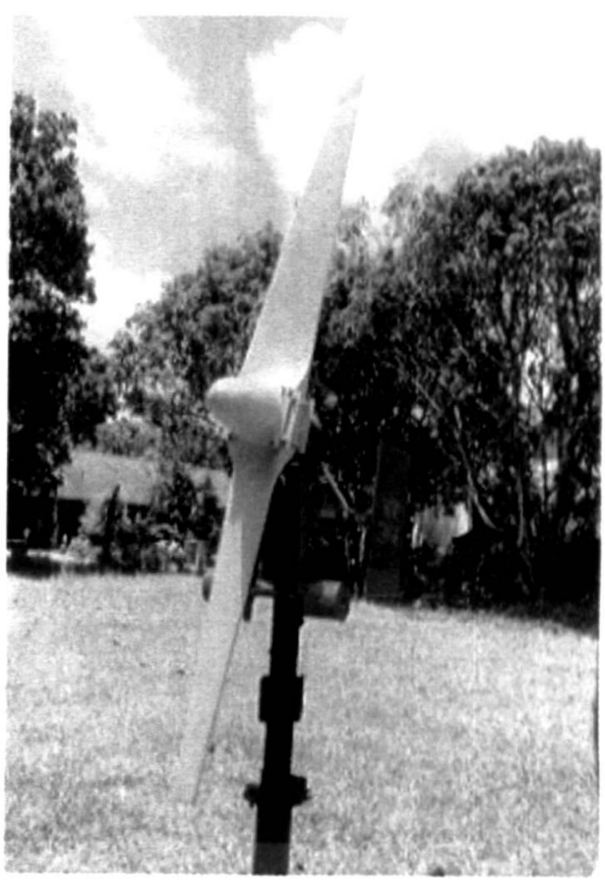

Figure 2 : picture of the tilt up type small wind turbine

Eng. Mahinsasa Narayana, B.Sc. Eng. (Moratuwa), AMIE (SL) MPhul (Eng) (Moratuwa). Mechanical Engineer, NERD Centre of Sri Lanka.

Eng. Kapila Peiris. B.Sc. Eng (Moratuwa), M phil (Agriculture Engineering), M.A. (Buddhist Studies), MIE (SL). C.Eng. (SL). Mechanu al Engineer, NERD Centre of Sri Lanka. 
A counter balancing weight or a spring mechanism is needed to build the restoring moment to hold the rotor to the wind [4]. Then wind rotor is tilted up ward due to the resultant torque of moment of the drag force of wind rotor and restoring moment of counter balancing weights around the pivoted point.

\section{Moments from D'Alambert's forces}

By using D'Alambert's principle a dynamic system can be converted into a static system. Then the system can be analysed by using static equilibrium conditions. In this case for each and every point forces of magnitude $\delta m_{1} f_{t}$ are applied in the opposite direction to its acceleration. $\delta m_{l}$ is mass at the point $i$ and $f i$ is acceleration at the point i. D'Alambert forces on the rotor, generator and counter weight are shown in the figure 3.

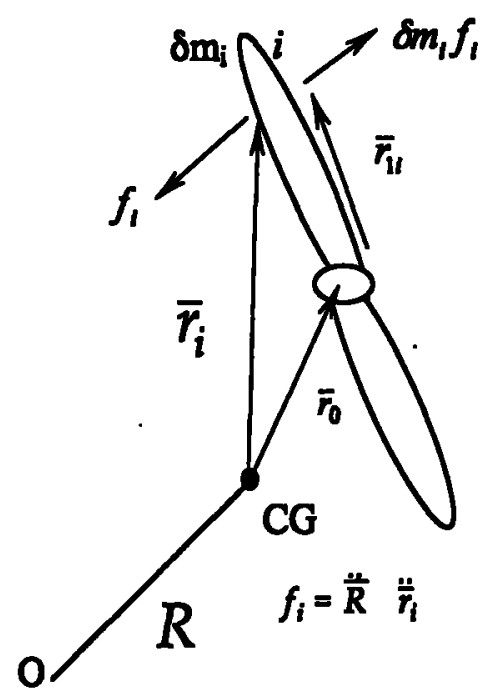

Figure 3 : D'Alambert forces on the rotor, generator and counter weight

Moments of D'Alambert forces on the rotor, generator and counter weight system about " $O$ ";

$$
\begin{aligned}
& M_{0}=\Sigma\left(\bar{R}+\bar{r}_{1}\right) \times \operatorname{dn} n_{1} \ddot{\bar{R}}+\ddot{\bar{r}} \\
& =\Sigma \delta m_{1}\left[\left(\bar{R}+\bar{r}_{i}\right) \times \ddot{\bar{R}}+\left(\bar{R}+\bar{r}_{i}\right) \times \ddot{\bar{r}}_{i}\right] \\
& \text { - } \Sigma \delta m_{1} \bar{R} \times \ddot{R}+\underbrace{\ddot{\bar{R}} \Sigma \delta m_{1} r_{1}} \\
& +\underbrace{\bar{R} \times \Sigma \delta m_{i}}_{0} \ddot{\bar{r}}_{i}+\Sigma \delta m_{1} \bar{r}_{i} \times \ddot{\bar{r}}_{1} \\
& M_{0}=m \bar{R} \times \ddot{\bar{R}}+\frac{\sum d\left(\bar{r}_{i} \times \delta m_{i} \dot{\bar{T}}_{i}\right)}{d t}
\end{aligned}
$$

Now $\quad(\dot{\theta} \gg \dot{\alpha}, \gamma)$ therefore $\dot{\bar{R}} \ll \dot{\bar{r}}_{i}$,
Velocity at point"i" $-\dot{\bar{R}}+\dot{\bar{T}}_{i}=\bar{V}_{i}$

$\therefore|\dot{\vec{V}}|_{1} \sim\left|\dot{\bar{r}}_{1}\right| \sim \theta\left|\bar{r}_{11}\right|$

$\dot{\boldsymbol{\theta}}=$ Angular speed of the rotor about rotor axis, $\bar{r}_{1}=\bar{r}_{0}+\bar{r}_{11}$

Now,

$$
\begin{aligned}
& \Sigma \bar{r}_{1} \times \delta m_{1} \dot{\bar{r}}_{1}-\Sigma \delta m_{1}\left(\bar{r}_{0}+\bar{r}_{11}\right) \times \bar{V}_{1} \\
& -\bar{r}_{0} \Sigma \underbrace{\delta m_{1} \bar{V}_{1}}_{.0}+\Sigma \delta m_{1} \bar{r}_{11} \times \bar{V}_{1}
\end{aligned}
$$

then, $\Sigma \delta m_{i} \bar{r}_{11} \times \bar{V}_{1}=\bar{I} \dot{\theta}$

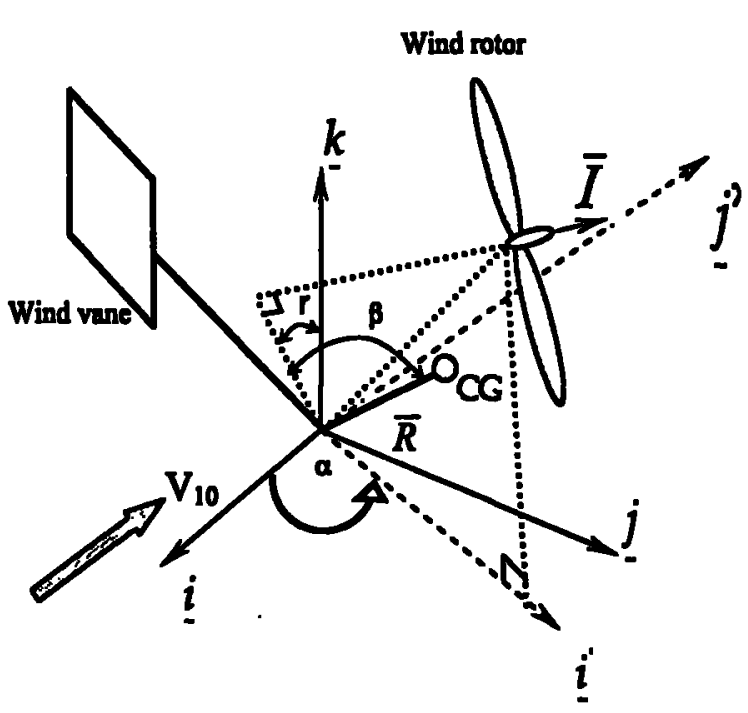

Figure 4 : Orientation of wind rotor and vane

Where, V1 - Velocity of wind, CG - Centre of gravity of rotor, generator \& counter weight

Therefore;

$M_{0}=m \bar{R} \times \ddot{\bar{R}}+\frac{d(\bar{I} \dot{\theta})}{d t}$

Where; $|\bar{l}|$-Moment of inertia of wind rotor about rotor axis, $\frac{\bar{I}}{|\bar{I}|}$-Unit vector along rotor axis,

m -Total mass of the rotor, generator \& counter weight,

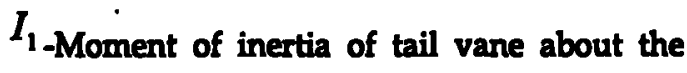
$\underline{k}_{\text {axis }}$

According to figure 4;

$\bar{R}=a \sin (\beta-\gamma) \cos \alpha \underline{i}+a \sin (\beta+\gamma) \sin \alpha \underline{j}$

$+a \cos (\beta-\gamma) \underline{k}$

$\bar{I}=|\bar{I}|[\cos \gamma \cdot \cos \alpha . \underline{i}+\cos \gamma \cdot \sin \alpha \cdot \underline{j}+\sin \gamma \cdot \underline{k}]$

Now, wind rotor, generator, counter weight system \& wind vane is free to move about $\underline{k}$ axis. 
Therefore,

$\underline{k}$ component of $\left\{\left[m \bar{R} \times \ddot{\bar{R}}+\frac{d(\bar{I} \dot{\theta})}{d t}\right]-I_{1} \ddot{\alpha}\right\}$

- $\left[\begin{array}{l}k \text { component of moment } \\ \text { generated by wind on vane \& rotor }\end{array}\right]$

Therefore;

$-m[a \sin (\beta-\gamma) \cos \alpha A-a \sin (\beta-\gamma) \sin \alpha B]$

$-I[\ddot{\theta} \sin \gamma+\dot{\theta} \dot{\gamma} \cos \gamma]-I_{1} \ddot{\alpha}=M_{k}$

$M_{k} \Rightarrow$ Moment due to aerodynamic forces on rotor and vane about $\underline{k}$ axis

Similarly rotor \& counter weight system is free to move about $j^{\prime}$ axis

Therefore,

$\underline{j}$ component of $\left[m \bar{R} \times \ddot{\bar{R}}+\frac{d(\bar{I} \dot{\theta})}{d t}\right]$

$=\left[\begin{array}{l}j \text { component of moment } \\ \text { generated by wind on vane \& rotor }\end{array}\right]$

$\sin \alpha\left\{\begin{array}{l}\left.\operatorname{asin}(\beta-\gamma) \sin \alpha\left[\ddot{\gamma} \sin (\beta-\gamma)-\gamma^{2} \cos (\beta-\gamma)\right]\right\}_{n} \\ -A a \cos (\beta-\gamma)\end{array}\right.$

$-\cos \alpha\left\{\begin{array}{l}\left.a \cdot \sin (\beta-\gamma) \cos \alpha \cdot \alpha\left[\gamma \sin (\beta-\gamma)-\gamma^{2} \cos (\beta-\gamma)\right]\right\}_{m} \\ -B \cdot a \cos (\beta-\gamma)\end{array}\right.$

$C-m g a \sin (\beta-\gamma)-M_{f}$

$M_{j} \Rightarrow$ Moment due to aerodynamic forces on

rotor about $\frac{j^{\prime}}{\text { axis }}$

where:

$A=\frac{d^{2}[a \sin (\beta-\gamma)]}{d^{2}} \sin \alpha$

$-\alpha\left[-\gamma \cos (\beta-\gamma) \sin \alpha-\dot{\gamma}^{2} \sin (\beta-\gamma) \sin \alpha\right.$

$-\dot{\gamma} \dot{\alpha} \cos (\beta-\gamma) \cos \alpha+\ddot{\alpha} \sin (\beta-\gamma) \cos \alpha$

$\left.-\dot{\alpha} \dot{\gamma} \cos (\beta-\gamma) \cos \alpha-\dot{\alpha}^{2} \sin (\beta-\gamma) \sin \alpha\right]$

$\bar{I}-|\bar{I}| \cdot[\cos \gamma \cdot \cos \alpha \underline{\underline{i}}+\cos \gamma \cdot \sin \alpha \cdot \underline{j}+\sin \gamma \underline{\underline{k}}]$

Now, wind rotor, generator, counter weight system \& wind vane is free to move about $\underline{k}_{\text {axis. }}$

$$
\begin{aligned}
& B=\frac{d^{2}[a \sin (\beta-\gamma) \cos \alpha]}{d^{2}} \\
& =\alpha\left[-\gamma \cos (\beta-\gamma) \cos \alpha-\gamma^{2} \sin (\beta-\gamma) \cos \alpha+\gamma \cos \sin \alpha \cos (\beta-\gamma)\right. \\
& \left.-\ddot{\alpha} \sin (\beta-\gamma) \sin \alpha+\dot{\alpha} \dot{d} \cos (\beta-\gamma) \sin \alpha-\dot{\alpha}^{2} \sin (\beta-\gamma) \cos \alpha\right] \\
& C=-\vec{p}[\ddot{\theta} \cos \alpha \cos \gamma+\dot{\theta} \dot{\alpha}(-\sin \alpha) \cos \alpha+\dot{\theta} \dot{\gamma}(-\sin \gamma)] \underline{I} \\
& +\cos \alpha[\ddot{\theta} \cos \gamma \sin \alpha+\dot{\theta} \gamma(-\sin \gamma) \sin \alpha+\dot{\theta} \dot{\alpha} \cos \gamma \cos \alpha] j
\end{aligned}
$$

\section{Aerodynamic forces on the wind rotor and vane}

\subsection{Aerodynamic forces on wind rotor}

An aerodynamic axial thrust force on the rotor is found out by considering the axial momentum of the flow through the rotor. In this study side thrust force is determined by considering wind rotor is a buff body.

$V_{2} / V_{1} \quad k$

where; $V_{1}$ - Upstream wind speed,

$V_{2}$ - Down stream wind speed,

$k$ - Axial induction factor

The elementary thrust force $(d F)$, can be determined by the general dynamics theory. Consider the axial momentum of the flow through the annulus:

Thrust $=$ (rate of mass flow, $m$, through the annulus of the thickness $d r$ ) $\times$ (change in the axial velocity).

Then, axial thrust on the annulus of $r_{r}$

$d F=r_{r} \pi \rho . d r_{r} V_{1}^{2}\left(1-k^{2}\right)$

$F_{t}=\frac{1}{2} V_{1}^{2} \operatorname{sR}_{r}^{2} \rho C_{i}$

$C_{t}=\frac{2}{R_{r}} \sum\left(1-k^{2}\right) r_{r} d r_{r}$

where; $R_{r}$-radius of wind rotor, $r_{r}$ - distance from centre of the rotor, drr-width of blade element,

$\rho$-density of air, Tip speed ratio $\left(\lambda_{0}\right)=\dot{R_{1}} / V_{1}$

Axial induction factors ( $k$ values) for each blade element (annulus) can be determined by comparing the expression for $d F$ \& $d M$ derived by blade elementary theory with that derived by general dynamics [2]. Therefore $k$ values for each tip speed ratio $\left(\lambda_{0}\right)$ can be calculated and then corresponding thrust forces were found.

$C_{1}$ is a function of $\lambda_{0}$. Then thrust force of the rotor at each rotor rotational speeds can be calculated for a known tip speed ratio $\left(\lambda_{0}\right)$.

$F_{1}=\frac{1}{2}\left(V_{1} \cos \alpha \cdot \cos \gamma\right)^{2} \pi_{r}^{2} \rho C_{1}$

Side thrust of wind rotor is calculated by considering as a buff body. Wind velocity component applied on the wind rotor is shown 
in figure 5. Aerodynamic forces $(F, \sin \Omega, F, C o s$ $\left.\Omega \& F_{1}\right)$ on the wind rotor are shown in figure 6. Then, side thrust force is $F_{;}$;

$F_{s}=\frac{1}{2} C_{d} V_{0}^{2} \rho A_{s}$

Where; $F$, -Side thrust, $C_{d}$-Drag coefficient for side thrust force of rotor, As - Effected rotor area to the side thrust

$V_{0}=V_{1} \sqrt{(\cos \alpha \sin \gamma)^{2}+\sin ^{2} \alpha}$

For a computation purposes, $F_{1}$ is divided into two components of $F_{s} \sin \Omega \& F_{z} \cos \Omega$ in the rotor plane.

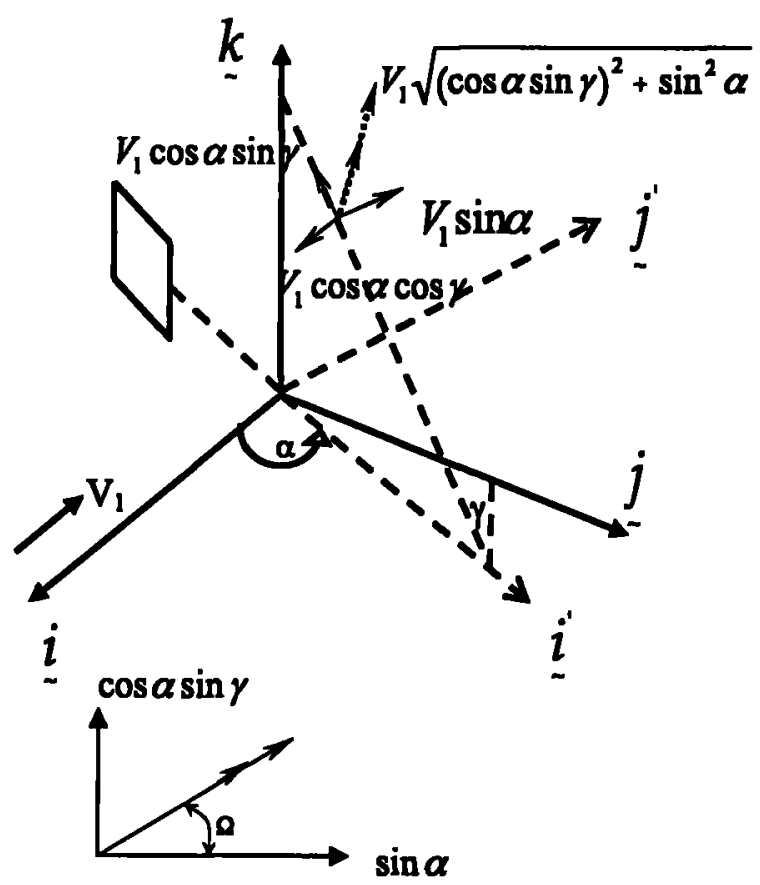

Figure 5 : Wind velocity component applied on the wind rotor

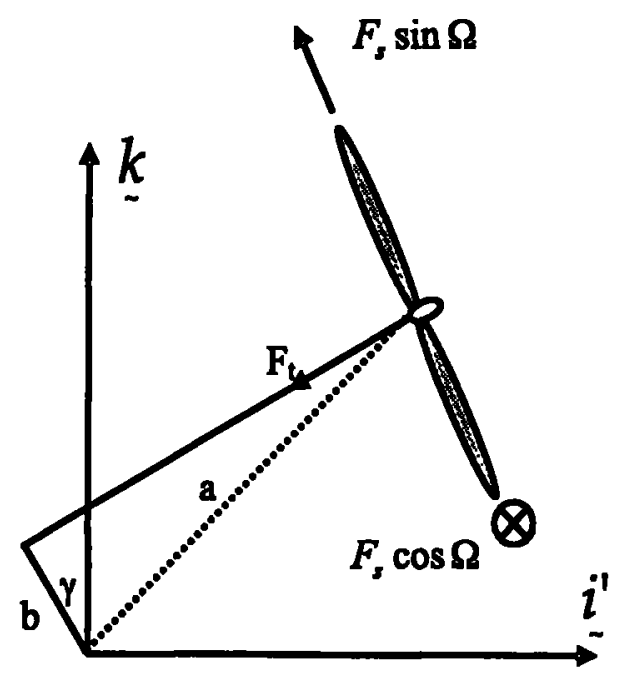

Figure 6 : Aerodynamic forces on the wind rotor
Then, axial thrust on the annulus of $r_{r}$

$$
\begin{aligned}
& d F=r_{r} \pi \rho \cdot d r_{r} V_{1}^{2}\left(1-k^{2}\right) \\
& F_{t}=\frac{1}{2} V_{1}^{2} \pi R_{r}^{2} \rho C_{t} \\
& C_{t}=\frac{2}{R_{r}} \sum\left(1-k^{2}\right) r_{r} d r_{r}
\end{aligned}
$$

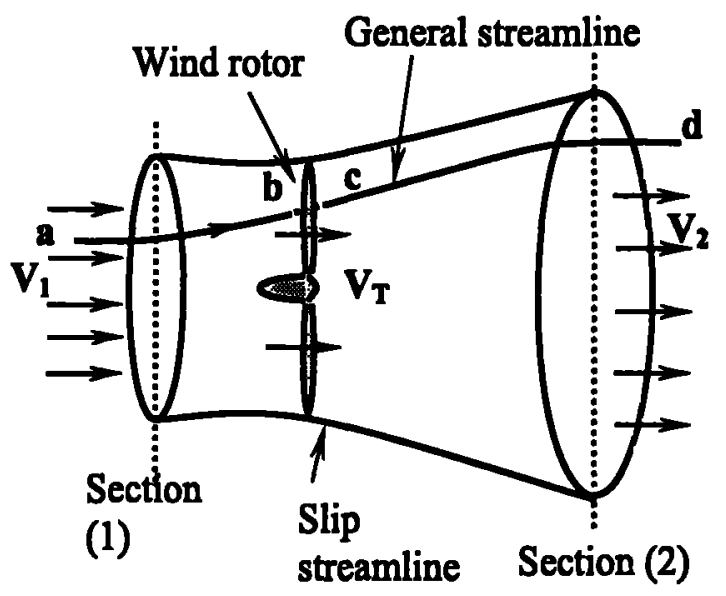

Figure 7 : Control flow volume used for wind rotor

where;

$R_{r}$ - Radius of wind rotor

$r_{r} \quad$ - Distance from centre of the rotor

$d r_{r}$ - width of blade element

$\rho$ - Density of air

Tip speed ratio $\left(\lambda_{0}\right)=\dot{\theta} R_{y} / V_{1}$

Axial induction factors ( $k$ values) of each blade elements (annulus) can be determined by comparing the expression for $d F \& d M$ derived by blade elementary theory with that derived by general dynamics [2]. Therefore $k$ values for various tip speed ratios $\left(\lambda_{0}\right)$ can be calculated and then corresponding thrust forces were found. Graphical presentation of thrust coefficients $\left(C_{k}\right)$ with each tip speed ratios $\left(\lambda_{0}\right)$ is shown in figure 8 .

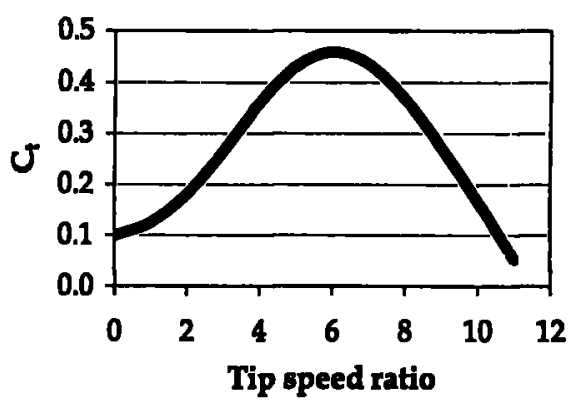

Figure 8 : Thrust coefficient of wind rotor 
$C_{1}$ is a function of $\lambda_{0}$. Then thrust force of each rotor rotational speeds can be calculated for a known tip speed ratio $\left(\lambda_{0}\right)$.

$F_{t}=\frac{1}{2}\left(V_{1} \cos \alpha \cdot \cos \gamma\right)^{2} \pi R_{r}^{2} \rho C_{t}$

Side thrust of wind rotor is calculated by considering as a buff body. Wind velocity component applied on the wind rotor is shown in figure 5. Aerodynamic forces $(F, \sin \Omega, F, \cos$ $\left.\Omega \& F_{2}\right)$ on the wind rotor are shown in figure 6 .

Then, side thrust force $F_{a}$ is

$F_{s}=\frac{1}{2} C_{d} V_{0}^{2} \rho A_{s}$

Where;

$F$, - Side thrust

$C_{d b}$ - Drag coefficient for side thrust force of rotor

A, - Effected rotor area to the side thrust

$V_{0}=V_{1} \sqrt{(\cos \alpha \sin \gamma)^{2}+\sin ^{2} \alpha}$

For a computation purposes, $F$ is divided into two component of $F_{\text {a }} \sin \Omega \& F_{3} \operatorname{Cos} \Omega$ in the rotor plane.

\subsection{Aerodynamic forces on wind vane}

In this wind turbine, a rectangular shape tail vane is used for yawing the wind turbine to correct orientation. Considering lift and drag forces can calculate aerodynamic force on the tail vane. Aerodynamic forces on wind vane are shown in figure 9. Assumed that lift and drag coefficients ( $C_{1}$ and $C_{d}$, respectively) for tail vane can be approximated by the "flat plate" equations (equation $9 \& 10$ ).

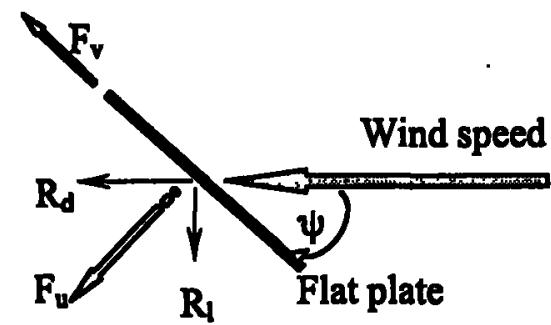

Figure 9 : Aerodynamic forces on tail vane

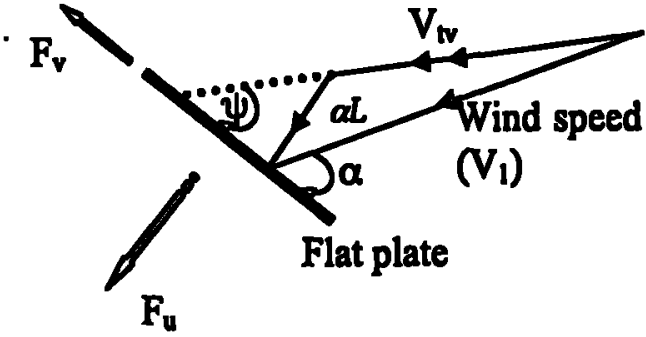

Figure 10 : Wind velocity diagram for tail vane

To determine the aerodynamic forces on the tail vane, wind speed relative to the tail vane should be found. In this calculation, the radial velocity of every point of the tail vane is taken as $\dot{\alpha} L$, since the width of tail vane is relatively small with comparing the length of the vane arm. According to the velocity diagram (figure 10), relative wind speed to the tail vane $\left(V_{v v}\right)$ can be derived as follows.

$V_{v t}=\sqrt{\left(V_{1}-\dot{\alpha} L\right)^{2}+\dot{\alpha} L \cos ^{2} \alpha}$

According to figure 10;

$\psi=\alpha-\tan ^{-1}\left[\frac{\dot{\alpha} L \cos \alpha}{V_{1}-\dot{\alpha} L \sin \alpha}\right]$

$V_{\mathrm{w}}$-Wind speed relative to the tail vane Flat plate equation

$\Psi$ - Angle of attack to the tail vane

$C_{l}=2 \sin \psi \cos \psi$

$C_{d}-2 \sin ^{2} \psi$

Then lift and drag forces on the wind vane can be calculated as follows;

$R_{l}=\frac{1}{2} \rho C_{l} V_{\text {ot }}^{2} A_{\text {v }}$

$R_{d}=\frac{1}{2} \rho C_{d} V_{v t} A_{v}$

Where;

$A_{v}$ - Area of the tail vane

L - Distance from centroid of the tail vane to the hinge axis.

$F_{u}-R_{l} \cos \psi+R_{d} \sin \psi$

$F_{v}=R_{l} \sin \psi-R_{d} \cos \psi$

Substitute the solution for $C_{1}, C_{d} R_{1} \& R_{d}$ to the equation (13).

$F_{u}-V_{v t}^{2} \rho A_{v}\left(\sin \psi \cos ^{2} \psi+\sin ^{3} \psi\right)$ 
4. Moments from aerodynamic forces on the wind turbine

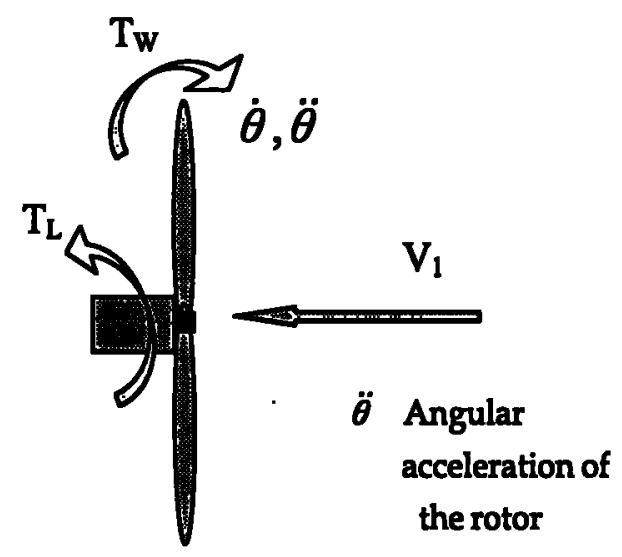

Figure 11 : Applied torques on the wind rotor

Generated aerodynamic torque by wind rotor is depend on the wind speed and rotational speed of the rotor. Torque characteristics $\left(C_{m}\right)$ of a wind rotor can be determined by considering geometrical parameters (blade angles and chord lengths) and characteristics of rotor profiles [3].

\section{$T_{W}(V, \dot{\theta})=$ Torque by wind}

Resistance torque of generator is rely on the rotational speed of the generator. Relevant resistant torques for each rotational speed (i) can be found by the torque characteristics of the generator.

$T_{L}(\dot{\theta})=$ Torque by load (Generator)

Then; $T_{W}-T_{L}=|\bar{I}| \ddot{\theta}$

Moment due to aerodynamic forces on rotor and vane about $\underline{k}$ axis;

$$
\begin{aligned}
& M_{\underline{k}}=a \sin (\beta-\gamma) F_{s} \cos \Omega+F_{u} L \\
& M_{\underline{k}}=\frac{1}{2} C_{d u} \cdot V_{0}^{2} \rho A_{s} \cos \Omega a \sin (\beta-\gamma) \\
& +L V_{v}^{2} \rho A_{v}\left(\sin \psi \cos ^{2} \psi+\sin ^{3} \psi\right)
\end{aligned}
$$

Substitute the solution of $F$, from equation 4 and the solution for $F_{\text {u }}$ from equation 5 .

Moment due to aerodynamic forces on rotor about j'axis

$$
M_{f^{\prime}}=a \cos (\beta-\gamma) F_{s} \sin \Omega+b F_{t}
$$

Substitute the solution of $F_{1}$ from equation 3 \& solution for $F_{s}$ from equation 4
$M_{L^{\prime}}=\frac{1}{2} C_{d d} V_{0}^{2} \rho \alpha_{d} \sin \Omega a \cos (\beta-\gamma)$

$+\frac{1}{2}\left(V_{1} \cos \alpha \cos \gamma\right)^{2} \pi R_{r}^{2} \rho C_{r} b$

From equation (1), (2) and (6) it is clear that, if $\mathrm{V}_{1}, \alpha, \dot{\alpha}, \gamma, \dot{\gamma} \& \theta$ are known at a particular instant (at the time $t_{0}$ ) then for that particular instant $\ddot{\alpha}, \ddot{\gamma} \& \ddot{\theta}$ could be determined. Now values of $\alpha, \dot{\alpha}, \gamma, \dot{\gamma} \& \dot{\theta}$ after a very small time interval of " $\delta t$ " could be determine by assuming linear relations within the time interval " $\mathrm{dt}$ ", now again $\ddot{\alpha}, \ddot{\gamma} \& \theta$ at time $t_{0}+\delta$ t could be found by equation (1), (2) and (6) with this iterative method it is possible to investigate the variation of kinematic parameters $\alpha, \dot{\alpha}, \ddot{\alpha}, \gamma, \dot{\gamma}, \ddot{\gamma}, \dot{\theta}, \& \ddot{\theta}$ of the system.

\section{Conclusion}

In this analysis D'Alambert's principle was used to derive the governing equations of the system. Therefore effects such as gyroscopic, centrifugal/ centripetal are not relevant in this study. D'Alambert's principle provides a method of analysing a dynamic system with any degree of complexity with a very simple fundamental approach.

Basic external forces applied to the tilt up type wind turbine are from wind. Aerodynamic forces on the wind rotor and tail vane were determined separately. In this study, the rotational speed of the rotor $(\dot{\theta})$ was assumed to be very high compared with the other angular movements $(\dot{\alpha} \& \dot{\gamma})$ of the system. In this context, to simplify the solutions, aerodynamic force on the rotor was divided into two categories such as axial thrust and side thrust. The axial thrust force was calculated by considering the wind speed component of the rotor axis and the side thrust force was calculated by considering wind rotor as a buff body. However, the blade elementary theory will give better and comprehensive results although the methodology is complex [1]. The developed model is to be tested in a wind tunnel. Then the model could be improved and also the comparison of practical results and predicted values from the model for various iterative time intervals " $(t$ " could be done. Finally with this model it would be possible to optimise the parameters of the system such as dimensions " $a$ ", " $b$ " (Figure 5) position \& mass of the counter weight in order obtain maximum power output from the wind rotor in varying wind speed condition. 


\section{References}

1. Ackerman M. C. Yaw Modelling of Small Wind Turbines, Journal of Wind Engineering and Industrial Aerodynamics, 39(1992) 1-9.

2. Gourieres, D Le. Wind Power Plants Theory and Design, Oxford: Pergamon press, 1982, p 76-120.
3. Narayana M \& Sugathapala A G T, Performance of Battery-Charging Wind Turbine Developed by The NERD Centre, The 10th Asian Congress of Fluid Mechanics 17-21 May 2004, Peradeniya, Sri Lanka

4. Narayana M, Gyroscopic Effect of Small Scale Tilt up Horizontal Axis Wind Turbine. World Renewable Energy Congress VI Brighton, UK. , 1-7, July 2000. 